\title{
Transparency in a Human-Machine Context: Approaches for Fostering Shared Awareness/Intent
}

\author{
Joseph B. Lyons and Paul R. Havig \\ Air Force Research Laboratory, 711 Human Performance Wing/Human Effectivess Directorate, \\ Wright-Patterson AFB, OH 45433 \\ \{Joseph.Lyons.6, Paul.Havig\} @us.af.mil
}

\begin{abstract}
Advances in autonomy have the potential to reshape the landscape of the modern world. Yet, research on human-machine interaction is needed to better understand the dynamic exchanges required between humans and machines in order to optimize human reliance on novel technologies. A key aspect of that exchange involves the notion of transparency as humans and machines require shared awareness and shared intent for optimal team work. Questions remain however, regarding how to represent information in order to generate shared awareness and intent in a human-machine context. The current paper will review a recent model of human-robot transparency and will propose a number of methods to foster transparency between humans and machines.
\end{abstract}

Keywords: transparency, human-machine interaction, trust in automation, trust.

\section{Introduction}

Robotic platforms have been projected to revolutionize the landscape of modern life with applications ranging from support of the elderly, customer service, education, rehabilitation, and medicine [1]. These systems may offer advantages over humanbased operators in situations where humans are not available, not capable, nor motivated to perform the necessary actions. Robotic platforms (also called autonomous systems within the Department of Defense, or DoD, vernacular) could be used in a military context to perform missions such as high-risk reconnaissance, search and rescue, logistics (e.g., transport), training, defensive security/crowd control, firefighting, and potentially kinetic operations. Full autonomy, where a system is permitted to execute complete decision and behavioral initiative, has yet to be realized for the vast majority of systems, though technology advances may enable greater autonomy in the future. Yet, despite technological advances in many areas related to robotics, humans maintain a critical role in executing supervisory control and serving as collaborators (some may suggest partners) with novel technology. The need for humans to be involved with robotic systems as controllers, supervisors, or teammates will not likely change in the near future, at least not for Department of Defense (DoD) -oriented technologies. Thus, it is critical that human-centric requirements be engineered into robotic platforms to encourage optimal use and reliance on newly developed systems. 
Much of the research in this area has focused on human-robot interaction (HRI) and has been driven in recent years by advances in commercial-off-the-shelf platforms, advances in the science of human-like robotic interfaces, and advances in the design of robotic platforms in terms of navigation and locomotion. A growing interest has emerged in understanding HRI using a more social orientation. There has been a movement toward considering robotic platforms as teammates rather than as tools [2]. Moving from tools to teammates requires that systems be designed with more naturalistic interaction styles which may attempt to leverage the nuances of human-human interactions. Regardless of the approach used, human resistance or acceptance of these systems will be a key factor in shaping the success or demise of robotic systems. "The human ability to accept robots into teams ultimately will determine the success or failure of robot teammates. Although the technical capabilities of robots to engage in coordinated activity is improving (as visible for robot-only teams at RoboCup), we believe humans' innate expectations for team-appropriate behavior pose challenges to the development of mixed teams that cannot be fixed with technology innovation." [3; p.486]. Research has consistently demonstrated that humans naturally anthropomorphize technology [4], and this phenomenon may be more prominent within an HRI domain as humans may seek to ascribe "intention" to robotic systems given their appearance and planned usage in socially-oriented contexts [2].

Human-human teams can be effective or not effective depending largely on a number of social elements including development of shared mental models [5], team processes (e.g., communication, conflict management, coordination [6]) and training [5]. Effective teamwork has a number of socio-emotional components including: shared goals, shared awareness, desire to be interdependent, motivation toward team goals, performance toward team activities, and trust among team members [3]. Many of these same principles will apply to a human-machine context, particularly when the system exhibits human characteristics, is used for social tasks, and is used in the context of uncertainty. One of the keys for optimizing the human-machine relationship will be to present the human partner with cues related to the team-orientation and mission-centric performance of the robotic system. Yet, this is easier said than done.

Autonomy for robots may include autonomous sensing, planning, or acting [7]. A robotic platform that is programmed to behave autonomously can face a number of "decision points" during dynamic environmental constraints. Complicating matters is the fact that many DoD missions could involve both high levels of uncertainty and threat, as well as time pressure. Robotic platforms in the commercial world could also include high-risk domains such as aviation or driving, both of which can involve lifethreatening consequences when errors occur. Robotic platforms could be used in tandem with manned platforms and the number of platforms on the battlefield could be considerable, placing high demands on the human's ability to monitor the battlefield [8]. The demands of monitoring the performance of multiple robotic systems could foster sub-optimal decision strategies, suggesting that systems be designed to present the right information at the right time without overloading their respective operator/teammate [8]. In order to address the dynamics of environmental changes, the nuances of various tasks, and the need for team-oriented social cues robotic systems should be designed to foster transparency. 


\subsection{Transparency in a Human-Machine Context}

Transparency in a human-robot context can be defined as a method to establish shared intent and shared awareness between a human and a machine [9]. The concept of transparency emerged from the trust in automation literature as way to reduce uncertainty regarding the performance and or behavior of an automated tool. Interestingly, providing information to human users of automation can also create greater uncertainty when done poorly (i.e., when overloading the users or when presenting users with irrelevant information). Therefore, the type and amount of information presented to users must be tailored to the unique situation in which the information is to be used. Prior research on trust in automation found that providing human operators with information related to the reliability of an automated tool promoted more optimal reliance strategies on the tool [10]. Further, information related to the limitations of an automated tool aids in trust recovery following errors of the automation [11]. This added information appears to be useful in deciphering the boundary conditions under which the tools are more or less capable. Thus, providing humans with information related to the performance of an automated tool appears to be beneficial. Kim and Hinds [12] applied a similar logic within a human-robot context and found that providing explanations of anomalous robotic behavior was related to more attributions of blame when the system made an error, though only when the system was described as having higher versus lower autonomy. The added information seems to add value for humans interacting with technology, perhaps because it offers clues regarding the performance of the system. Further, this information seems to be more important when the system has greater control over its actions (i.e., when it is more autonomous). This is logical given that performance is the strongest predictor of trust within a human-robot context [13]. The competence (i.e., ability) of a person is also a critical driver of interpersonal trust; however, so is the benevolence and integrity of a person [14]. Given the social-emotional engagement that is possible between humans and robotic platforms and the social nature in which robotic platforms may be used, it is plausible that both performance-oriented and socially-oriented transparency factors matter within a human-robot scenario.

In this spirit, Lyons [9] outlined a novel model of human-robot transparency involving an intentional model (including social intent and purpose), task model, analytical model, environment model, teamwork model, and human state model. The intentional model highlights the overall purpose of the system and provides cues to the human regarding the social intent of the system. The task model may include an understanding of the particular task (perhaps via a cognitive task analysis or a task analysis), information that communicates the robot's goals, information that characterizes the robot's progress in relation to the goals, information to communicate awareness of capabilities in relation to the goals, and finally communications that signify awareness of performance/errors in relation to the goals. The analytic model should communicate the decision logic used by the robot to initiate action. The environment model should communicate awareness of constraints present within the mission context (e.g., weather, relevant variations in terrain, threat information, etc.). The teamwork model should visualize the division of labor between the partners in real-time, 
adjusting to the dynamic nature of tasks as activities as traded off between partners. Finally, the human state model should display relevant metrics related to the human's performance (i.e., workload, stress, performance trends) in the human-robot context. The notions of shared intent and shared awareness are key objectives related to transparency. Thus, the methods for fostering transparency are clustered around each of these higher-level domains of shared intent and shared awareness. Below we outline some initial thoughts we have on how HRI should evolve for effective teaming. Note that an interesting research question is the amount of anthropomorphizing one may (or may not) want to incorporate in a human-machine team. These ideas are put forward as an attempt to get the community to discuss how this teaming should most effectively be instantiated and then eventually tested.

Shared Intent. From the perspective of functionality, appearance seems to matter quite a bit in a human-robot context. Appearance of a robot may confer functionality to the humans interacting with it based on expectations [1; 15]. Some of these expectations conform to gender roles. Research by Eyssel and Hegel [16] compared robots with typical male features such as short hair to robots with female features such as long hair and found that "male" robots were perceived as being more agentic than females and more suitable for masculine tasks such as security or repairs. In contrast, "female" robots were perceived as more warm, better suited for care giving, and service relative to "males". Further, research by Broadbent [17] found that robots with a human-like appearance were perceived as being more amicable, social, and having the most personality relative to robots that were less human-looking. The critical point from a transparency perspective is that the desired functionality of the system should map directly into its appearance as much as possible.

In addition to the function-orientation of the intention model, a robot also needs to confer social intent to its human teammate. Rich social information exchanges may enhance human-robot interactions as this added information could serve as a useful cue to the human representing the system's understanding, engagement, and intent [18]. The social style of the system will be an important factor in determining the "intent" of the system (as perceived by the human) which will likely have significant implications for one's trust of the system. Given the uncertainties inherent to humanrobot interactions, understanding the social intent of a system will help humans to anticipate "why" a robot took a particular action, which is equally as important as understanding what the action was. Further, understanding robot intent will allow the human to anticipate a range of actions given particular environmental constraints. The latter would be useful for DoD operations since the DoD operates with a number of guidelines/rules for conflict (e.g., the Law of Armed Conflict) and understanding social intent, which may include adherence to a set of ethical - albeit - programmed principles of a robotic platform, will aid human operators and partners in making informed decisions regarding how much behavioral initiative to provide to the robotic system (see [19] for a review). Understanding the drivers of robotic behavior could also be important for autonomous systems used in the commercial domain. For instance, it will be important for humans to understand the driving etiquette of autonomous cars, particularly during congested traffic, inclement weather, or urban routes 
involving high pedestrian traffic. This information will help humans to anticipate the behavior of the robotic platforms. Social cues can also provide humans with rich information regarding how the robot "views" their human teammate [20]. Akin to human-human social exchanges, it is often not what is said but how it is said that shapes one's reactions to others. These social cues could represent concepts such as benevolence, social etiquette, and team-oriented communication.

Shared Awareness. Many human-robot interaction scenarios involve collocation between the human and the system. However, many DoD-relevant scenarios will involve distributed interactions, and having shared awareness in the latter context will be critical to ensuring optimal HRI. The transparency model by Lyons [9] outlines a number of awareness-based requirements between humans and robotic platforms. First, the robot should communicate knowledge about the task. The robot should communicate what task, or which step embedded within a series of tasks, it is currently working on. Inputs for this task model could originate from a task or cognitive analysis, which would specify the facets of each task to be accomplished. The robot should communicate progress within the task, and where possible identify when it has encountered obstacles that prevent task completion. The system should also communicate the decision logic used to initiate behaviors. This will be particularly useful when a behavior violates the expectations of the human. A key element of distributed teaming between humans and robotic platforms will be developing a shared awareness of the environmental constraints within a task domain. This has been shown to be important for deep space missions where humans cannot be collocated with robotic platforms [7]. Finally, the robotic platform should communicate awareness of the distribution of labor in team-based tasks, and an awareness of human state characteristics such as workload and stress. In the section below, the authors outline a few high-level ideas for creating these transparency facets within a HRI context.

\section{Interface Techniques}

\subsection{Shared Intent}

The importance of shared intent cannot be overlooked if trying to form an effective Human-Robotic team. It is clear from watching team failures in any environment (e.g., sports, work, familial, etc.) that the potential for miscommunication and misunderstanding easily arises when the intent of all players is ambiguous. The same will likely hold true for human-machine partnerships. In a DoD context, perhaps the most important sharing of intent would be when the robot has to update the human partner as to why it deviated from the original plays/programming (i.e., what was the intent of the change and what are the follow-on consequences). This is especially important if there is a reliance on the human to help form a new course of action. Shared intent can flow from the appearance, social cues, and prescribed behavioral repertoire of the system. From a non-DoD perspective, shared intent would be extremely helpful in Human-Robotic teams for disaster relief. Robotic systems have been used to survey damage associated with events such as the Tsunami that hit Japan in 2011 and the 
super typhoon that hit the Philippines in 2013. If the systems were given the mission of finding individuals in need following these natural disasters they would need to be flexible in their planned routes. When the systems had the decision initiative to deviate from flight plans, they humans interacting with them would need to understand the intent of the system or else the system would run the risk of being overridden by the human. Take the example of plan deviation; it would be important for the intent to be communicated quickly and accurately so the human partner could make a more effective decision.

From an appearance standpoint, researchers have demonstrated that the form of a robotic system tends to shape the human expectations associated with the system [1]. Thus, the key for establishing transparency in purpose is to align the appearance with the desired function. The more socially-oriented the desired function, the more anthropomorphic the system should be designed. Robots intended for mechanistic functions such as loading/unloading, transport, assembly may not need anthropomorphic features as much as those engaging in social-oriented activities. Robots designed for security purposes may want to incorporate features that are useful for deterrence such as size or the presence of a weapon to shape perceptions of relative formidability [21]. Spider legs, relative to regular legs or tracked capabilities, have also been shown to be associated with higher perceived aggressiveness in prior research [22]. More research is needed in this area to better understand how different robotic forms relate to human expectations.

Non-verbal cues from robotic systems can be used to decipher intent from the systems. Social cues like gaze can signal attention, facial expressions can be used to communicate emotion, and action can represent intention [20]. Gaze seems to foster greater recall of information when humans interact with robotic instructors [23]. Gaze may help to establish shared attention and it may help to foster social norms or social etiquette within a task context. Gaze may be particularly useful in situations where the human and the system must exchange a lot of information. For instance, service robots taking orders for merchandise or food may be equipped with gaze features to demonstrate engagement with a particular person. Exploring social etiquette within an automated scenario, Parasuraman and Miller [24] found that automated tools were more effective when they avoided interrupting humans during tasks and when they used a polite versus impatient style. Social etiquette between humans and robotic systems may benefit from having an established social etiquette that is appropriate for the particular task context in question. It may also be imperative for the social etiquette to evolve as the dynamics of a situation change. For instance, it is possible that when a robot encounters a time-sensitive constraint the humans will respond better to etiquette that reflects this temporal demand and urgency.

The communication exchanges between humans and robots will also serve as a key indicator of robot intention. Communications that exemplify the notion of benevolence, the belief that a trustor has the trustee's best interest in mind [14], may promote a human's perceived benevolence from the robot when the robot suggests a change of behavior is needed. Robots could use phrases such as, "My recommended course of action is in your best interests based on my assessment of your goals." That phrase communicates an awareness of the human's needs and that the suggested action would be the best course of action in relation to those needs. Robotic communications 
may also influence human perceptions of their competence. The use of specific versus more general nouns has been shown to be related to greater perceived persuasion [23]. Also, robots tend to be perceived as having greater expertise when they use a combination of vocal variability, gaze, and gestures [23]. Other research has found that robot-expressed emotions are more easily perceived by humans when multiple information channels are used at once (e.g., gestures coupled with expressions) [25]. Thus, the use of social cues and corresponding behavior may provide information to the human about the capabilities and intent of the robot.

Understanding the social intent of robotic systems will also involve the concept of robot ethics. Researchers discussed the idea of ethical governors for robotic systems [19]. Such systems may identify the thresholds for risk based on established conventions in warfare - this could incorporate a decision logic tree for behaviors. Presenting this logic to humans may provide useful information pertaining to the social intent of the robot. This could also be represented via robotic emotional displays where the robot interface could portray particular emotions in contexts where it might be expected to display emotions such as guilt [19]. Expressed guilt would signify that the system is aware that it has violated the desired state of the human. Emotion expressions, even digital emotional displays, signify an awareness of consequences and thus one can infer intent from them [26]. In fact, it is often the extrapolation of intent through an emotional appraisal process that drives human behavioral reactions to agent-based emotional displays [26]. Thus, the idea of robot "intent" will be fundamental in predicting how humans respond to robotic social cues. Designers need to be cognizant of such effects lest their systems fall victim to misaligned perceptions among user communities. The issue of alignment between perceived intent (i.e., appraisals of intent [26]) and robotic emotional displays will increase with importance as robots gain more behavioral and decision authority, and as robots are used in more team-based scenarios.

\subsection{Shared Awareness}

Prior research has outlined a number of recommendations for the design of automated tools: it should be aware of progress towards goals, suggest actions that support goals, be inquisitive when uncertainty is high, communicate intentions and limitations, and serve as a collaborator [27]. Many of these concepts are consistent with the idea of the task model of transparency. Robots could use a real-time indicator within a humanmachine interface to signify which step in a particular task, or set of tasks, that a robot is currently working on. Simple process flow diagrams could be used to represent a set of tasks and as the robot completes one step and moves on to the next step a realtime indicator could be used to track progress within the process map. When the robot encounters an obstacle or is otherwise unable to complete a task it should communicate this limitation/constraint to the human, and where possible share the reason for the failure. Geo-registering could be used to track the location of distributed platforms. In the event of a disturbance in signal, which is possible in military domains characterized by Anti-Access Area Denial (A2AD) domains, the interface could provide a range of possible locations based on historical waypoints. The showing of robotic moves/interactions on a geospatial world view would be a most logical step 
for the majority of current concepts for HRI. If the true concept of autonomous vehicles of the future is "autonomous action" then the human needs only be a passive viewer of the work (i.e., the human is on-the-loop as opposed to being in-the-loop) and would only need to intercede when there is an operationally relevant teaming decision to be made. However, simple route information may not be enough. In current military operations, it is up to the pilot to pay attention to things such as fuel, time-on-station, sensor capabilities, and then make the decision through the operations floor if a change in plans is required based on the mission goals. In this future scenario, an automated system may be capable of monitoring its fuel status for example, but may not have the ability to effectively hand-off operations to another autonomous vehicle. In this case, the human operator would have to intervene and re-route the appropriate asset. In such situations, it would be optimal for the human to have complete knowledge of the "health" of the system and so the aforementioned characteristics (e.g., fuel) would need to be depicted and/or reported in some way that is actionable by the human. An initial design concept could involve creating a display to represent the health status on any platform similar say to the status board one may have in a hospital setting. The hospital metaphor is important as the most important health information should be highlighted in some logical manner so the human can more quickly assess the situation and make a decision.

Understanding the analytical underpinning of a robot during a performance context is also very important. Human collaborators need to understand the logic tree for the system [7] particularly when the system evidences some anomalous behavior. For instance, when the human expects the platform to be in location A and it emerges in location $\mathrm{C}$, it needs to immediately explain the anomalous behavior to the human partner. In this situation, it would be best for the robot to report changes in a clear and logical manner that allows the human to quickly re-assess the situation and prepare for new operations. One way this could be done is to have the robot report with a quick verbal protocol as to when the mission changed, why it changed, and what was accomplished due to the change (or what was not) accomplished due to the change. After this quick debrief then, depending upon the mission and thorough explanation, using the original planning interface would then help in the re-planning process. The idea of the quick debrief is to get the human on the same page as the robot much in the same way a human would do when reporting back to a commanding officer (i.e., quick debrief then details as needed). The importance of understanding the logic driving behavior increases as systems gain initiative to execute mission objectives. In other words, the greater autonomy afforded to a robotic system, the greater the need for the human to understand the robot's decision logic driving its behavior.

As robots are used in novel domains they are certain to encounter unexpected constraints within the environment. These constraints may come in the form of difficult weather, terrain obstructions, or even hostile threats within military operations. Robotic interfaces should be designed to foster shared awareness between the human and robot regarding any environmental changes that may influence mission effectiveness. This shared awareness could be enabled through the use of sensors on the platform to gauge weather patterns, as well as cameras to display physical constraints and threats in the area.

Finally, robots need to communicate an awareness of the division of labor when engaging in team-based tasks with human partners. Using the same notion of a 
process map or task analysis described above, the interface could represent which activities were under the robot's control and which were the responsibility of the human. Taxonomies for representing different levels of automation such as those outlined in [28] could be used to signify the degree of autonomy currently allocated to the robot for a particular task. This could be particularly useful when a human is collaborating with more than one robotic platform and is responsible for supervising and adjusting them within an operational context.

\section{Conclusion}

The future of HRI and design approaches to foster transparency within this domain is ripe for future research. Indeed, there is considerable human-human teaming research on-going that can be leveraged as the HRI moves forward, and this has been evidenced by the growth of social robotics. The key evolution in recent years is the consideration of the robot as a partner not a thing, tool, or vehicle. Once a true teaming concept is accepted and adopted, our supposition is that the interface will follow naturally though the HCI of implementing effective transparency methods aiming to engender shared intent and shared awareness between the human and his/her robotic teammate.

\section{References}

1. Veloso, M., Aisen, M., Howard, A., Jenkins, C., Mutlu, B., Scassellati, B.: WTEC Panel Report on Human-Robot Interaction Japan, South Korea, and China. World Technology Evaluation Center, Inc., Arlington (2012)

2. Ososky, S., Schuster, D., Phillips, E., Jentsch, F.: Building appropriate trust in humanrobot teams. In: Proceedings of AAAI Spring Symposium on Trust in Autonomous Systems, pp. 60-65. AAAI, Palo Alto (2013)

3. Groom, V., Nass, C.: Can robots be teammates? Benchmarks in human-robot teams. Interaction Studies 8(3), 483-500 (2007)

4. Nass, C., Moon, Y.: Machines and mindlessness: Social responses to computers. J. of Social Issues 56, 81-103 (2000)

5. Salas, E., Cooke, N.J., Rosen, M.A.: On teams, teamwork, and team performance: Discoveries and developments. Human Factors 50(3), 540-547 (2008)

6. Cohen, S.G., Bailey, D.E.: What makes teams work: group effectiveness research from the shop floor to the executive suite. J. of Management 23, 239-290 (1997)

7. Stubbs, K., Wettergreen, D., Hinds, P.J.: Autonomy and common ground in human-robot interaction: A field study. IEEE Intelligent Systems, 42-50 (2007)

8. Chen, J.Y.C., Barnes, M.J., Harper-Sciarini, M.: Supervisory control of multiple robots: Human performance issues and user interface design. IEEE Transactions on Systems, Man, and Cybernetics - Part C: Applications and Reviews 41(4), 435-454 (2011)

9. Lyons, J.B.: Being transparent about transparency: A model for human-robot interaction. In: Proceedings of AAAI Spring Symposium on Trust in Autonomous Systems, pp. 48-53. AAAI, Palo Alto (2013)

10. Wang, L., Jamieson, G.A., Hollands, J.G.: Trust and reliance on an automated combat identification system. Human Factors 51, 281-291 (2009) 
11. Dzindolet, M.T., Peterson, S.A., Pomranky, R.A., Pierce, L.G., Beck, H.P.: The role of trust in automation reliance. Int. J. of Human-Computer Studies 58, 697-718 (2003)

12. Kim, T., Hinds, P.: Who should I blame? Effects of autonomy and transparency on attributions in human-robot interactions. In: Proceedings of the 15th International Symposium on Robot and Human Interactive Communication (RO-MAN 2006), pp. 80-85. IEEE, Hatfield (2006)

13. Hancock, P.A., Billings, D.R., Schaefer, K.E., Chen, J.Y.C., Ewart, J., Parasuraman, R.: A meta-analysis of factors affecting trust in human-robot interaction. Human Factors 53(5), 517-527 (2011)

14. Mayer, R.C., Davis, J.H., Schoorman, F.D.: An integration model of organizational trust. Academy of Management Review 20, 709-734 (1995)

15. Fischer, K.: How people talk with robots: Designing dialogue to reduce user uncertainty. AI Magazine, 31-38 (2011)

16. Eyssel, F., Hegel, F.: (S)he's got the look: Gender stereotyping of robots. J. of App. Soc. Psych. 42(9), 2213-2230 (2012)

17. Broadbent, E., Kumar, V., Li, X., Sollers, J., Stafford, R.Q., MacDonald, B.A., Wegner, D.M.: Robots with displays screens: A robot with more humanlike face display is perceived to have more mind and a better personality. PLoS ONE 8(8), e72589 (2013)

18. Breazeal, C., Aryananda, L.: Recognition of affective communicative intent in robot directed speech. Autonomous Robots 12(1), 83-104 (2002)

19. Arkin, R.C., Ulam, P., Wagner, A.R.: Moral decision-making in autonomous systems: Enforcement, moral emotions, dignity, trust, and deception. Proceedings of the IEEE 100(3), 571-589 (2012)

20. Coradeschi, S., Ishiguro, H., Asada, M., Shapiro, S., Thielscher, M., Breazeal, C., Mataric, M., Ishida, H.: Human-inspired robots. IEEE Intelligent Systems 21(4), 74-85 (2006)

21. Fessler, D.M.T., Holbrook, C., Snyder, J.K.: Weapons make the man (larger): Formidability is represented as size and strength in humans. PLoS ONE 7(4), e32751 (2012)

22. Sims, V.K., Chin, M.G., Sushi, D.J., Barber, D.J., Ballion, J., Clark, B.R., Garfield, K.A., Dolezal, M.J., Shumaker, R., Finkelstein, N.: Anthropomorphism of robotic form: A response to affordances? Proceedings of the Human Factors and Ergonomics Society Annual Meeting 49, 602-605 (2005)

23. Mutlu, B.: Designing embodied cues for dialogue with robots. AI Magazine, 17-30 (2011)

24. Parasuraman, R., Miller, C.: Trust and etiquette in high criticality automated systems. Communications of the ACM 47, 51-55 (2004)

25. Zecca, M., Mizoguchi, Y., Endo, I.F., Kawabata, Y., Endo, N., Itoh, K., Takanishi, A.: Whole body emotion expressions for KOBIAN Humanoid Robot: Preliminary experiments with different emotional expression patterns. Paper presented at the 18th IEEE International Symposium on Robot and Human Interactive Communication. Toyama, Japan (2009)

26. de Melo, C., Carnevale, P., Read, S., Gratch, J.: Reverse appraisal: The importance of appraisals for the effect of emotion displays on people's decision-making in a social dilemma. In: Proceedings of the 34th Annual Meeting of the Cognitive Science Society, Sapporo, Japan (2012)

27. Geiselman, E.E., Johnson, C.M., Buck, D.R.: Flight deck automation: Invaluable collaborator or insidious enabler? Ergonomics in Design: The Quarterly of Human Factors Applications 21, 22-26 (2013)

28. Parasuraman, R., Sheridan, T.B., Wickens, C.D.: A model for types and levels of human interaction with automation. IEEE Transactions on Systems, Man, and Cybernetics-Part A: Systems and Humans 30, 286-297 (2000) 